\title{
Neural signatures of model-free learning when avoiding harm to self and other
}

Patricia L. Lockwood ${ }^{\mathrm{a}, \mathrm{b}^{\star \wedge}}$, Miriam Klein-Flügge ${ }^{\mathrm{a}, \mathrm{b}^{\star \wedge}}$, Ayat Abdurahman ${ }^{\mathrm{a}, \mathrm{b}}$, \& Molly J. Crocketta, ${ }^{\mathrm{a}}$

aDepartment of Experimental Psychology, University of Oxford, Oxford OX1 3PH, United Kingdom

bWellcome Centre for Integrative Neuroimaging, Department of Experimental Psychology, University of Oxford

'Department of Psychology, Yale University, New Haven, CT, 06511, USA

${ }^{*}$ Correspondence should be addressed to:

Patricia L. Lockwood, Experimental Psychology, Tinsley Building, University of Oxford, OX1 3SR, United Kingdom. E-mail: patricia.lockwood@psy.ox.ac.uk

Miriam C Klein- Flügge, Experimental Psychology, Tinsley Building, University of Oxford, OX1 3SR, United Kingdom. E-mail: miriam.klein-flugge@psy.ox.ac.uk

Molly J. Crockett, Department of Psychology, 2 Hillhouse Avenue, New Haven, CT 06511, USA. E-mail: molly.crockett@yale.edu

NIndicates joint first authorship 


\section{Abstract}

3 Moral behaviour requires learning how our actions help or harm others. Theoretical

4 accounts of learning propose a key division between 'model-free' algorithms that 5 efficiently cache outcome values in actions and 'model-based' algorithms that 6 prospectively map actions to outcomes, a distinction that may be critical for moral 7 learning. Here, we tested the engagement of these learning mechanisms and their 8 neural basis as participants learned to avoid painful electric shocks for themselves 9 and a stranger. We found that model-free learning was prioritized when avoiding harm 10 to others compared to oneself. Model-free prediction errors for others relative to self 11 were tracked in the thalamus/caudate at the time of the outcome. At the time of choice, 12 a signature of model-free moral learning was associated with responses in subgenual 13 anterior cingulate cortex ( $\mathrm{sgACC}$ ), and resisting this model-free influence was 14 predicted by stronger connectivity between sgACC and dorsolateral prefrontal cortex. 15 Finally, multiple behavioural and neural correlates of model-free moral learning varied 16 with individual differences in moral judgment. Our findings suggest moral learning 17 favours efficiency over flexibility and is underpinned by specific neural mechanisms. 
A central component of human morality is a prohibition against harming others ${ }^{1,2}$. People readily avoid actions that might harm another person ${ }^{3-7}$, and this basic harm aversion is so strong that many people even find it distressing to perform pretend harmful actions, such as shooting someone with a fake gun ${ }^{8}$. Harm aversion is disrupted in clinical disorders such as psychopathy that have a strong developmental component ${ }^{9}$, and although harm aversion is robust in healthy adults, anyone who has watched young children fighting over a coveted toy knows that such an aversion is not present from birth. Indeed, a large literature documents the emergence of moral conduct over the course of development ${ }^{10,11}$. Cross-cultural differences in morality suggest moral behavior is fine-tuned to local environmental demands ${ }^{12}$, and lab experiments demonstrate how individuals can quickly adapt moral behavior to changing norms ${ }^{13,14}$. All this evidence highlights a critical role for learning in the development of harm aversion and moral behaviour more broadly ${ }^{6}$.

Recent work in computational neuroscience has advanced our knowledge of how organisms learn the value of actions and outcomes via reward and punishment ${ }^{15,16}$. An important theoretical distinction has been made between 'model-based' and 'model-free' learning 17,18. The computationally expensive model-based system builds an internal model of the environment and selects actions by prospectively searching the internal model for the best course of action ${ }^{19,20}$. The computationally efficient model-free system assigns values to actions through trial-and-error and selects actions retrospectively based on these 'cached' values; because it does not store a model of the environment, it can sometimes make sub-optimal recommendations (e.g., in settings where typically valuable actions lead to bad outcomes, or vice versa). These two systems are somewhat neurally dissociable, with model-based learning preferentially engaging lateral prefrontal cortex (LPFC), posterior parietal cortex and caudate $^{20-22}$ and model-free learning preferentially engaging putamen ${ }^{23,24}$, although both systems update their representations via prediction errors encoded in overlapping regions of ventral striatum ${ }^{20}$. Model-based and model-free systems often make similar recommendations about which actions are more valuable, but when they 
conflict, an arbitration process allocates control between them $22,25,26$. However, despite extensive theorizing that the model-based/model-free distinction may help to characterize puzzling features of moral learning and decision-making ${ }^{3,27-29}$, it remains unknown whether the moral consequences of actions affect the balance between model-based and model-free control, and whether common or distinct neural processes are engaged when learning to avoid harmful outcomes to self and others.

Past work on the neural basis of moral decision-making provides support for competing hypotheses. On the one hand, the sophistication of human morality seems to demand the kinds of complex representations afforded by model-based learning, suggesting learning to avoid harming others may preferentially engage the modelbased system. Supporting this view, people are easily able to learn to avoid harmful actions without directly experiencing their outcomes through trial and error, as may be required for model-based learning $3,27,30$. Moreover, moral decision-making in healthy adults consistently engages brain regions most strongly associated with the modelbased system, including lateral prefrontal cortex (LPFC), caudate, and temporoparietal junction (TPJ) $23,25,31$. Deciding to follow moral norms like fairness and honesty, and enforcing those norms on others via costly punishment, engages LPFC ${ }^{32-37}$, and disrupting LPFC function reduces moral norm compliance and enforcement ${ }^{38,39}$. During decisions to avoid harming others, LPFC encodes the blameworthiness of harmful choices and modulates action values in caudate and thalamus ${ }^{4}$, two subcortical areas shown to play a critical role in associative learning and pain processing as well as moral decision-making ${ }^{40-45}$. TPJ has been implicated in sophisticated representations of others' mental states and integrating these into social decisions ${ }^{46-48}$.

On the other hand, one principal function of model-free learning is to cache value in actions that are reliably adaptive, sacrificing flexibility for efficiency. Given that harming others is typically prohibited, actions that harm others may represent a special class of actions that are prioritized for model-free learning, similar to how certain classes of stimuli, like snakes and spiders, are "prepared" for aversive classical conditioning 49.

81 In other words, since avoiding harm to others is hugely important for social life, 
82 learning processes that fast-track harm-avoidant action selection to a habitual, 83 automatic process may be socially adaptive. Supporting this view, recent work 84 suggests that morality constrains mental representations of what actions are 85 considered possible; harmful actions are removed from choice sets as a default ${ }^{50}$, and choices that harm others are slower than helpful choices, suggesting an automatic tendency to avoid harm 5,51,52. Furthermore, recent studies of model-free learning to gain rewards for oneself and others have highlighted a distinct encoding of prediction errors concerning others' outcomes in the subgenual anterior cingulate cortex $(\mathrm{sgACC})^{53,54}$, a region that has been implicated in social and moral decision-making more broadly ${ }^{54-58}$. Model-free processes that distinguish learning about how one's actions affect others could provide a neural mechanism for prioritizing model-free learning in moral contexts.

To test these competing hypotheses, we used computational modelling and $\mathrm{fMRI}$ to probe the relative balance between model-based versus model-free processes, and their neural bases, when people learn to avoid moderately painful electric shocks for themselves and a stranger. After undergoing a pain thresholding procedure (see Methods), participants ( $\mathrm{N}=36$ ) completed a hybrid version of two paradigms previously proposed to reliably dissociate model-free versus model-based learning (Figure $1)^{20,22,31}$. We optimized the task in a way that allowed us to address the specific hypotheses examined in the present study (see Supplementary Note on Experimental Design for details). 

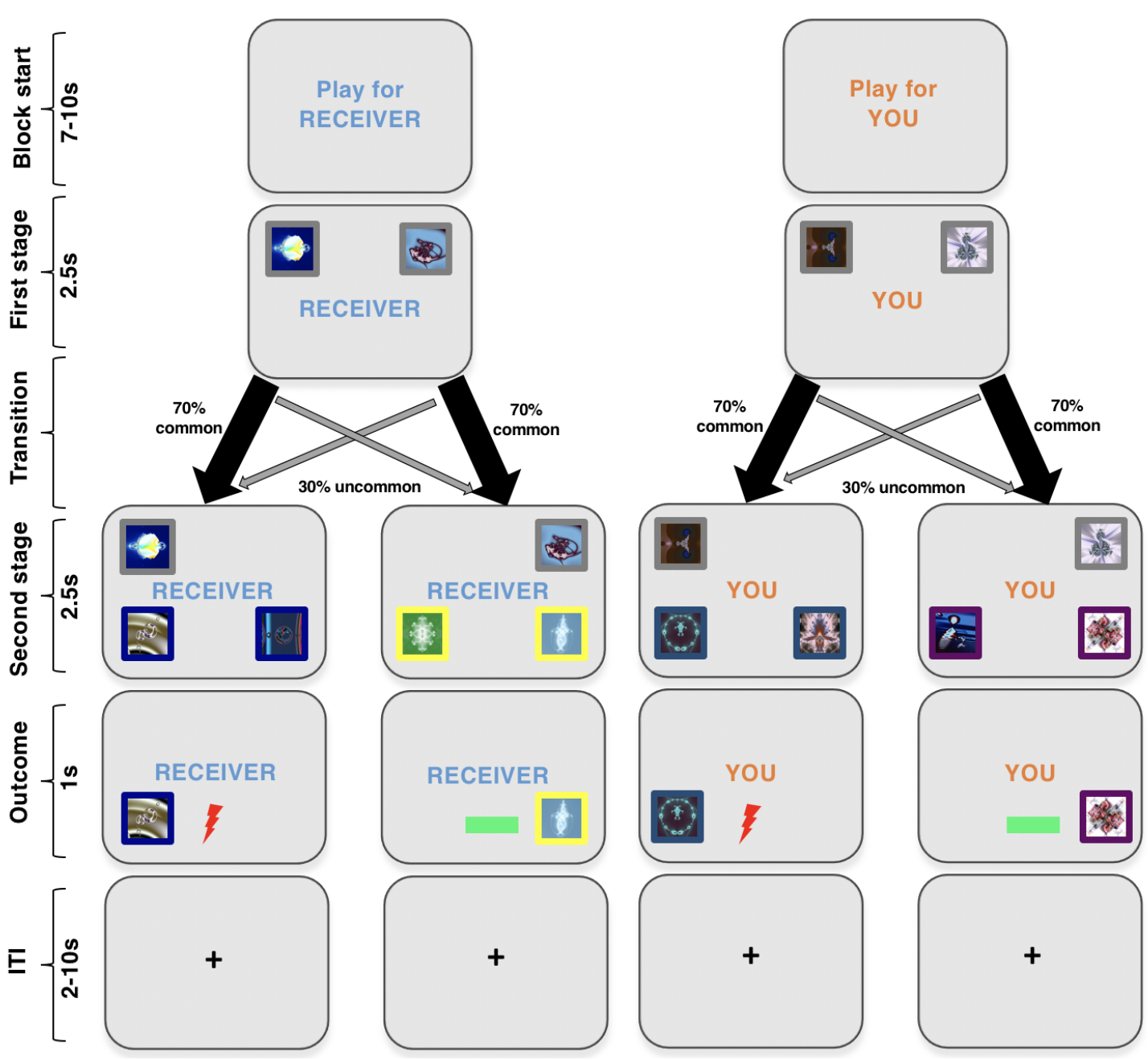

Figure 1 - Model-free and model-based aversive learning task. Participants completed a two-stage decision-making task to assess the tendency to engage in model-free and model-based learning. The task was a hybrid of two tasks previously shown to assess model-free and model-based learning processes 20,26 to probe learning to avoid aversive (shock) outcomes for either oneself or another person (the 'receiver', referred to as 'other' hereafter). At the beginning of each block an instruction cue signalled the recipient of the outcome (self or other). At the first stage two images were displayed that probabilistically led to one of two states depicted by different colours surrounding the boxes. In this example, to 'blue zone' or 'yellow zone' for the other participant, and 'turquoise zone' or 'purple zone' for self. Participants then made a second choice between two pictures in the coloured zone which was followed by an outcome of shock or no shock. The probability with which the boxes at the second stage delivered a shock or no-shock outcome drifted throughout the experiment (bounded between $0 \%$ and $100 \%$ with a drift rate of 0.2 ) and participants were instructed to keep learning throughout. $10 \%$ of the total electric shocks accumulated in the 'self' condition were delivered to the participant themselves at the end of the experiment whilst $10 \%$ of the electric shocks accumulated in the 'other' condition were

Results 
126 Participants completed a two-step decision-making task to index model-free and

127 model-based learning strategies (Figure 1). Prior to scanning, participants were

128 trained on the transition structure of the task using stimuli different from the main

129 experiment which allowed them to learn the probabilistic transition structure. The two-

130 step task distinguishes model-free and model-based learning by measuring people's

131 choices to stay or switch based on the outcome of the previous trial and the transition

132 structure. Theoretically, a purely model-free learner would ignore the transition

133 structure and repeat first-stage choices if they prevented pain on the previous trial but

134 switch choices if the previous choice caused pain. Thus, model-free learning is

135 reflected in a main effect of outcome (pain versus no pain) on subsequent first-stage

136 choice behaviour. In contrast, a model-based learner would take the transition

137 structure into account. While behaviour on common transitions would be similar for a

138 model-free agent, after rare transitions, a model-based agent would repeat a first-

139 stage choice if the outcome was pain, but switch if the outcome was no pain. Thus,

140 model-based learning is reflected in an interaction between outcome (pain versus no

141 pain) and transition (common versus rare). It is now well established that people

142 display a combination of model-free and model-based learning strategies when

143 learning about rewarding outcomes $20,31,59$ and initial evidence indicates that the same

144 is true for learning about aversive outcomes for oneself 60,61 . We therefore first

145 examined whether participants displayed a combination of model-free and model-

146 based processes during aversive learning for oneself and others, as observed in these

147 previous studies.

149 We performed a logistic regression analysis predicting first-stage stay versus switch

150 choices as a function of the outcome (pain or no pain), transition on the previous trial

151 (common or rare) and recipient (Self vs. Other). We included all main effects and

152 interactions in the model. We found a significant main effect of outcome $(t(35)=4.618$,

$153 p<.001$, confidence interval $(\mathrm{Cl})$ for beta estimate: $0.17,0.42$, Cohen's $d=0.77$ )

154 indicating a contribution of model-free learning, but also a significant transition by

155 outcome interaction $(t(35)=-3.173, p=.003, \mathrm{Cl}$ for beta estimate: $-0.23,-0.05, d=-$

156 0.53), indicating the presence of model-based learning (Figure 2a,b). This 
demonstrates that similar to reward learning, aversive learning is underpinned by a mixture of model-based and model-free processes.

160 Intriguingly, we also found a significant interaction between outcome (the model-free contribution to learning) and recipient, showing that people were more model-free for others relative to self $(t(35)=-2.31, p=.027, \mathrm{Cl}$ of beta estimates $-0.12,-0.008, d=-$

163 0.39). By contrast, there was no interaction between recipient and transition $x$ outcome, the model-based component of learning $(t(35)=-.459, p>.65, \mathrm{Cl}$ of beta estimates: $-0.071,0.045, d=-0.08)$.

We validated these behavioural results by repeating our analysis using the Ime4 package in $\mathrm{R}$ which ensured we had good estimates of random effects and accounted for variability in behaviour using Bound Optimization by Quadratic Approximation (see Methods). Our model predicted the tendency to switch vs. stay including all main effects and interactions and a random effect of subject, glmer(Switch_Stay NoPain_Pain*Transition*Agent+(1ISubject). All results remained the same (main effect of outcome (model-free) $Z=5.949, p<.001$; Outcome $x$ transition interaction (model-based) $Z=5.484, p<.001$; outcome $x$ recipient $Z=-2.154, p=0.031$; [outcome $\mathrm{x}$ transition (model-based)] $\mathrm{x}$ recipient $\mathrm{Z}=0.696, p=0.4864$ ). Again, these findings support the idea that people were more model-free when avoiding harming 177 others.

To further examine which outcomes most influenced the outcome $\mathrm{x}$ recipient

180 interaction, we performed separate post-hoc tests on the percentage of stay/switch choices for self and other following (a) only pain outcomes and (b) only no pain outcomes. This showed that the recipient difference was driven by increased switching after pain outcomes for other versus self $(p=.028)$, with no difference between recipients in the proportion of stay/switch choices after no pain outcomes $(p=.552)$.

185 This is important because the specificity of the effect rules out that people were simply more indifferent or inattentive to the outcomes of others compared to self. 

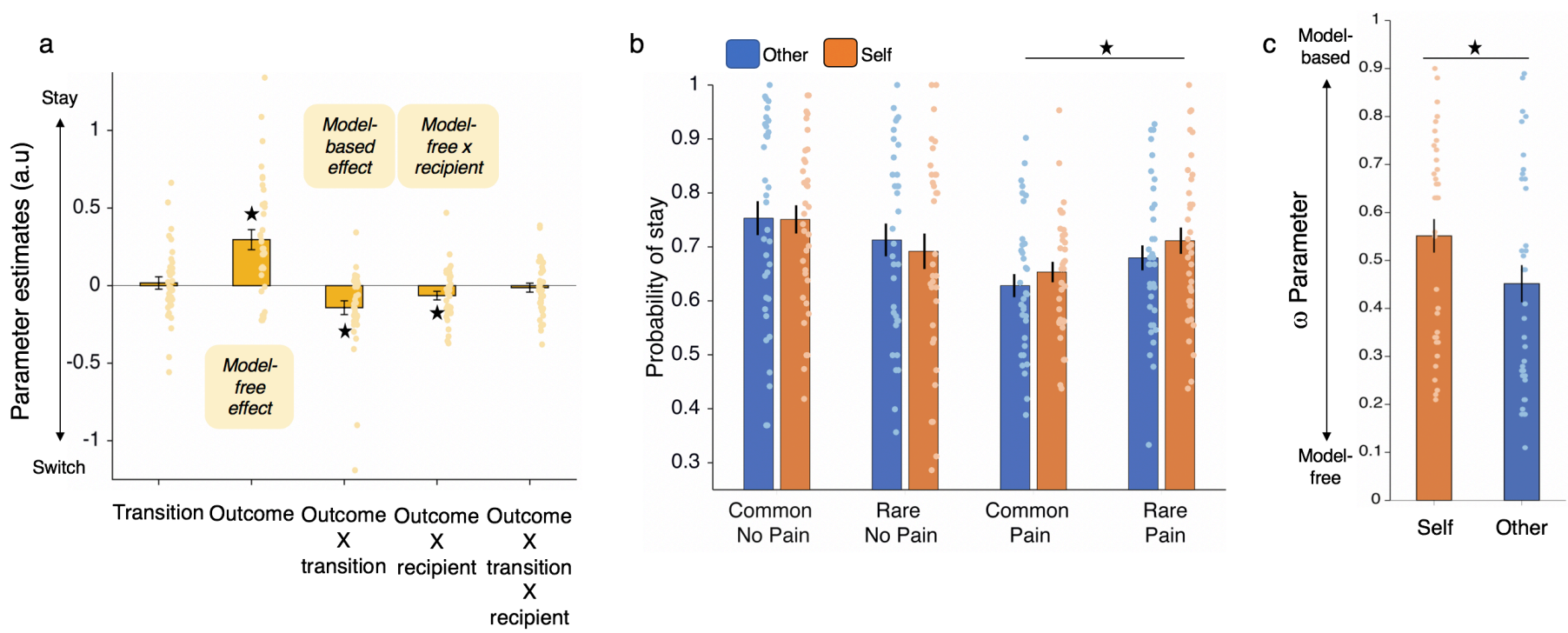

Figure 2 - Model-free and model-based choices when avoiding harming oneself and others. (a) Logistic regression coefficients predicting first-level stay/switch choices (mean \pm SEM). Participants exhibited a main effect of staying after no pain which indicated model free behaviour $(t(35)=4.618$, $p<.001$, confidence interval $(\mathrm{Cl})$ for beta estimate: $0.17,0.42$, Cohen's $d=0.77$ ) and a outcome by transition interaction which indicated model-based behaviour $(t(35)=-3.173, p=.003, \mathrm{Cl}$ for beta estimate: $-0.23,-0.05, d=-0.53)$. Intriguingly there was also a outcome $\mathrm{x}$ recipient interaction $((t(35)=-$ 2.31, $p=.027$, Cl of beta estimates $-0.12,-0.008, d=-0.39$ ) showing that participants were more modelfree, and thus more likely to switch after pain and stay after no pain, independent of transition type, when making choices for another person. (b). The probability of repeating a choice at the first-level ('stay') is plotted as a function of the transition and outcome on the previous trial. This shows that the outcome $x$ recipient interaction in (a) is mostly driven by fewer stay trials after pain, regardless of transition (two rightmost blue vs orange bars). Thus, the more pronounced model free behaviour for others is mostly driven by a lower probability of staying after pain outcomes rather than a higher probability of staying after no pain outcomes). (c) $\omega$ estimates from the best fitting model showed that the $\omega$ parameter was significantly lower for other $(0.45)$ than self $(0.55)$ consistent with the regression analyses that showed people were more model free when avoiding harm to others compared to self $(p<.02)$. Asterisks indicate significant difference at $p<.05$.

\section{Computational modelling of aversive learning for self and other}

209 Next, we fitted several trial-by-trial computational models to our data to examine

210 further which model best captured the described behaviours during aversive learning

211 for self and other. Deriving such trial-by-trial estimates that capture individual choice

212 preferences was a prerequisite for modelling the $\mathrm{fMRI}$ data and allowed us to support

213 our logistic regression analyses. We started with the full seven-parameter model

214 proposed by Daw and colleagues ${ }^{20}$ and compared this model to similar models with

215 fewer parameters (four of five) following modifications similar to those suggested in

216 previous studies (e.g. ${ }^{62,63}$ ); for details, see Methods and Supplementary Table 2).

217 We also included variants of the same models that involved separate learning rates 
218 for pain and no pain outcomes, given evidence suggesting differential learning as a

219 function of outcome valence (e.g. $\left.{ }^{64,65}\right)$. All of these models were initially fitted

220 separately on self and other blocks.

We found that a five-parameter model best explained behaviour compared to all alternative models tested. This model included separate learning rates for no pain and pain outcomes ( $\alpha$ Pain, $\alpha$ NoPain), a single temperature parameter capturing choice randomness $(\beta)$, a perseverance parameter capturing a tendency to stick with the previously made choice $(\rho)$ and a model-free/model-based weighting parameter $(\omega)$. Importantly, this five-parameter model best explained behaviour in both the self and other blocks (Supplementary Table 2).

We next compared the different estimated parameters for the self and other blocks.

231 This analysis showed a significant difference between the conditions in both the

232 perseverance parameter $\rho(t(35)=2.41, p=.02$,$) and the model-free/based weighting$ 233 parameter $\omega(t(35)=3.10, p=.0039$; Supplementary Table 3). To compare these 234 parameters more robustly, we then used maximum a-posteriori estimation performed 235 on the pooled data of self and other blocks to compare three models, one with 236 separate perseverance $\rho$ and $\omega$ parameters for self and other (and thus a total of seven parameters), one with separate $\omega$ parameters for self and other (and therefore a total of six parameters), and the original 5 parameter model (which assumes the same $\rho$ and $\omega$ across self and other blocks). We used these models to examine whether differences in the $\rho$ and $\omega$ parameters between self and other when fitted separately reflected true differences in the weighting of these parameters in a model comparison. This analysis showed that the model with separate $\omega$ 's for self and other, but not separate $\rho$ 's, best explained the data. Importantly, these $\omega$ parameters were also significantly different from one another (self $\omega=0.55$, other $\omega=0.45, t(35)=2.41$, $p=.02, d=0.40$, Figure 2c and Supplementary Table 4 and 5). Thus, consistent with the regression based behavioural analyses that did not rely on a computational model (Figure 2a), participants were more model-free than model-based when learning to avoid harming others, compared to self (Figure 2c). 


\section{Subcortical areas distinguish model-free prediction errors for self and other}

Previous neuroimaging studies of model-based and model-free reward learning have reported model-free prediction error signals in ventral striatum ${ }^{20}$. We therefore first sought to replicate this effect in our aversive learning paradigm. To facilitate comparison with previous studies of reward learning, no-pain outcomes were coded as 1 and pain outcomes coded as -1 . Therefore, a positive prediction error represents unexpected pain relief/avoidance, and a negative prediction error represents unexpected pain.

We built a general linear model (GLM1) that contained onsets for the first stage choice, second stage choice and outcome separately for self and other trials. These three time periods were each associated with parametric modulators from our winning model. These included the value difference between the two options at the first stage choice; the state prediction error based on the transition at the second stage choice; and the model-free prediction error at the time of the outcome. We focused our analysis on model-free prediction errors at the time of the outcome for two reasons. Firstly, our behavioural effects showed that self/other differences in learning emerged for modelfree but not model-based learning. Secondly, model-free and model-based prediction errors are highly correlated and careful examination of their separate influences has shown that they are both encoded in ventral striatum ${ }^{20}$.

272 We began our analyses by examining whether previously reported neural correlates

273 of value difference and state prediction errors were also observed in our novel paradigm. Several areas tracked inverse value difference and thus showed larger responses for choices that had a smaller value difference between the two first-stage options, including the most dorsal parts of anterior cingulate cortex near pre-SMA, bilateral inferior parietal cortex and middle frontal gyrus (Supplementary table 1), regions previously associated with the tracking of inverse subjective value difference $66-68$. These signals did not differ between self and other (see

280 Supplementary Table 1). Also consistent with previous findings ${ }^{21}$, we found evidence of a main effect for state prediction errors at the second stage in dorsal ACC ( $x=-6$, 
$282 \mathrm{y}=10, \mathrm{z}=52, \mathrm{Z}=4.85, \mathrm{~K}=906, \quad p<.001 \mathrm{FWE}$-corrected) that again showed overlap

283 between self and other (see Supplementary table 1).

Next, we tested whether model-free prediction errors were present in ventral striatum, as reported in a previous study examining model-based and model-free reward learning for self ${ }^{20}$ and several studies of reward based reinforcement learning 69 . We found a large bilateral cluster signalling prediction errors of harm avoidance (positive for no pain, negative for pain) in ventral striatum (right $x=10, y=12, z=-4, k=236$, $Z=5.84$, left $x=-16, y=6, z=-10, k=458, z=5.77, p<.05$ FWE-whole brain corrected after initial thresholding at $p<.001)$. Again, this signal did not significantly differ for self and other conditions (Right $t(34)=-.14, p=.89$, Left $t(34)=-1.47, p=.152)$ ).
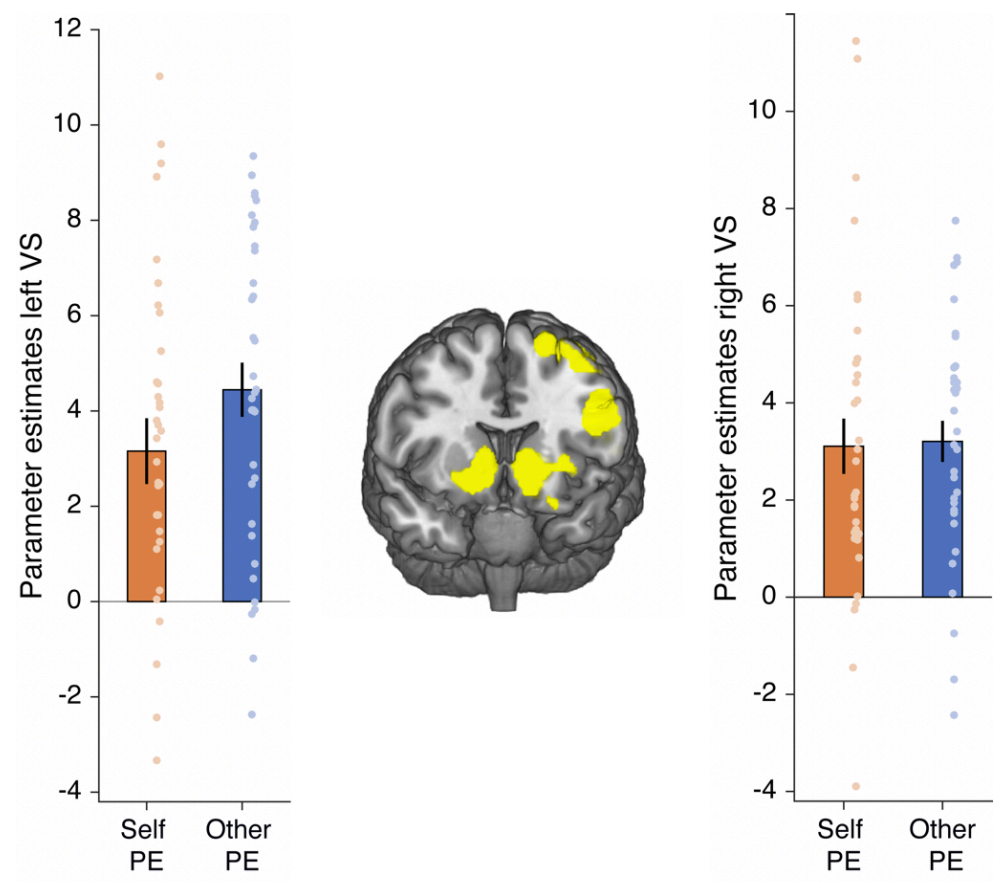

Figure 3 - Ventral striatum encodes prediction errors of pain avoidance for self and other. Ventral striatum (right $x=10, y=12, z=-4, k=236, z=5.84$, left $x=-16, y=6, z=-10, k=458, z=5.77, p<.05$ FWE-whole brain corrected after initial thresholding at $p<.001$ ) tracked model-free prediction errors for both self and other bilaterally, with no significant differences between conditions.

300 Given that our behavioral results indicated model-free learning was prioritized when avoiding harm to others (relative to self), we next sought to identify areas that distinguished model-free prediction errors for others (relative to self). This analysis revealed a cluster in the thalamus extending into the caudate $(x=16, y=-18, z=0$, 

pain avoidance when learning for other $(t(33)=2.30, p=.028)$ and negatively tracked prediction errors of pain avoidance when learning for self $(t(33)=-2.89, p=.007)$. Although this cluster extended into the caudate, the caudate ROI itself was not significant $(x=18, y=6, z=0, Z=3.49, k=2, p=.064$, FWE-SVC after initial thresholding at $p<.001)$.

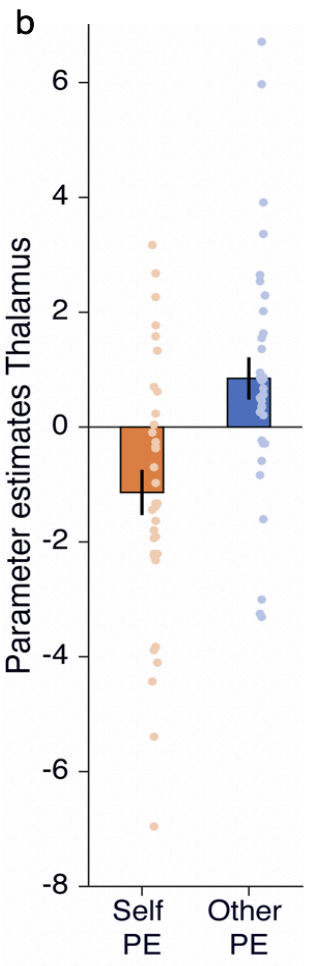

313 Figure 4 - Thalamus/caudate signal distinguishes model-free prediction errors for avoiding harm to other vs. self. (a) Thalamus cluster from the contrast other prediction error $>$ self prediction error $(x=16, y=-18, z=0, Z=3.50, k=84, p=.033$, FWE-SVC after initial thresholding at $p<.001)$ overlaid on an anatomical scan to show the extent of activation. (b) for illustration, parameter estimates extracted from the thalamus cluster are shown separately for self and other PE.

\section{Signatures of model-free influence are encoded in sgACC and TPJ}

One signature of model-free learning is a tendency to repeat previously rewarded actions and avoid previously punished actions, regardless of experienced transitions ${ }^{20}$. Such a model-free influence is thought to emerge at the time of choice by activating the reinforcement histories of potential actions and driving selection of the most valuable action in terms of its recent history ${ }^{70}$. In the context of our task, 
326 through model-free influence, an action that was unpunished on the previous trial

327 should be prioritized for selection ('stay'), while an action that was punished on the

328 previous trial should be avoided ('switch'). Importantly, because model-free learning

329 is insensitive to task structure, this process should occur regardless of whether the

330 transition from the first to the second stage experienced on the previous trial was

331 common or rare.

333 Therefore, to probe the neural signatures consistent with a model-free influence at the 334 time of choice, and any potential differences between self and other conditions, we examined neural responses during 'switch' and 'stay' choices at the first stage as a function of the outcome on the previous trial (no pain or pain). We created an additional GLM (GLM2) that modelled the onset of self trials after pain, self trials after no pain, other trials after pain, and other trials after no pain, with stay $(-1)$ and switch (1) coded as parametric modulators of each of these onsets. Thus, our analysis examined differential neural encoding of stay vs. switch decisions on the current trial, as a function of the outcome on the previous trial (pain or no pain) and its recipient (self or other).

344 Our analysis revealed a signal in sgACC consistent with a model-free influence on 'other' trials ( $\mathrm{x}=-2, \mathrm{y}=36, \mathrm{z}=6, \mathrm{~K}=498 ; \mathrm{Z}=3.88, p=.028$, FWE whole-brain corrected). This region was more active during stay relative to switch choices on the current trial, following a 'no pain' outcome on the previous trial, selectively in the 'other' condition (see Figure 5a-b and Supplementary Analysis for more details). Corroborating the view that this signal reflects a model-free influence, responses in sgACC were positively associated with the model-free $\mathrm{x}$ recipient interaction in behaviour $(r(33)=$ .36, $p=.04$, Figure $6 \mathbf{a}, \mathbf{b})$ such that participants with the largest sgACC difference between stay and switch following no pain for other also showed the strongest prioritization of model-free learning for others relative to self.

We observed the inverse pattern (i.e., opposite to what would be predicted by modelfree influence) in right TPJ ( $\mathrm{x}=54, \mathrm{y}=-38, \mathrm{z}=34, \mathrm{Z}=3.56, \mathrm{~K}=39, p=.03$, FWE-SVC). This region was more active during switch relative to stay choices on the current trial, 
bioRxiv preprint doi: https://doi.org/10.1101/718106; this version posted July 29, 2019. The copyright holder for this preprint (which was not certified by peer review) is the author/funder, who has granted bioRxiv a license to display the preprint in perpetuity. It is made available under aCC-BY-NC-ND 4.0 International license.

a

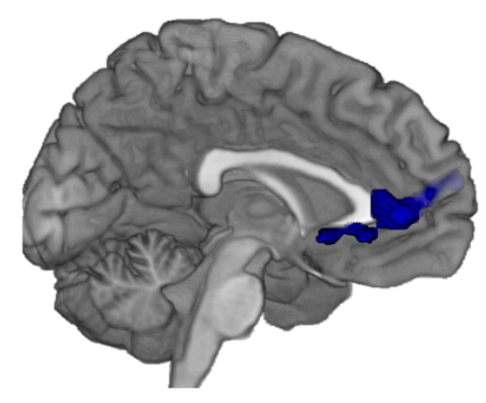

C

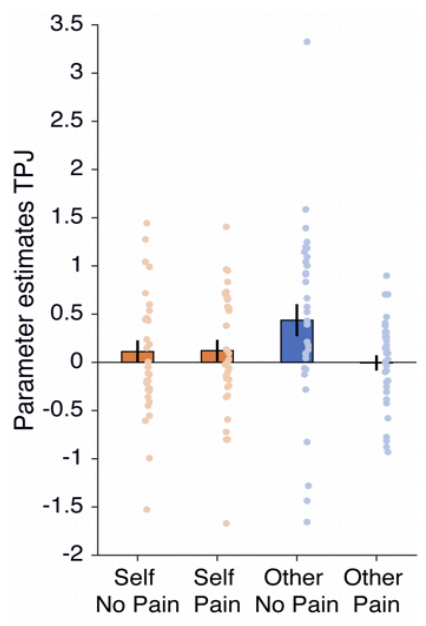

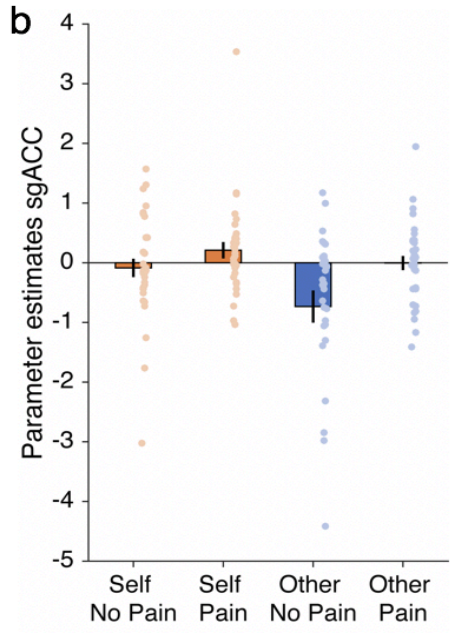

d

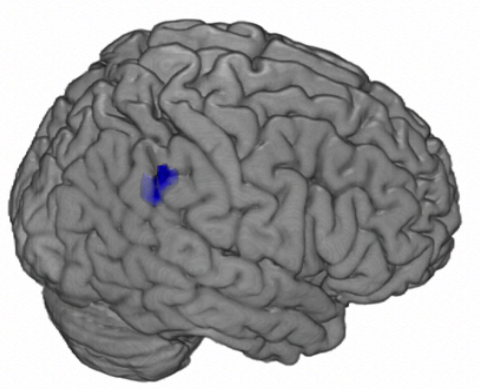

Figure 5 - Subgenual anterior cingulate cortex (sgACC) and temporo-parietal junction (TPJ) differentially encode signatures of model-free influence. (a,b) sgACC response $(x=-2, y=36, z=6$, $\mathrm{K}=498, p=.028$, FWE whole brain corrected, after initial thresholding at $p<.001$ ) to stay vs. switch after no pain for other overlaid on the medial surface of an anatomical scan. The observed BOLD pattern is consistent with model-free behaviour. (c,d) Temporoparietal junction (TPJ) ( $x=54, y=-38, z=34, Z=3.56$, $p=.03$, FWE-SVC after initial thresholding at $p<.001$ ) tracks switch more than stay after no pain for other. Cluster overlaid on an anatomical scan. 
375 Behavioral analyses indicated that participants on average showed a mixture of

376 model-based and model-free strategies, but prioritized model-free learning when

377 avoiding harm to others. Thus, we next sought to identify regions that might modulate

378 the model-free effects observed specifically in the 'other' condition in sgACC (Figure

379 6a). We therefore conducted psycho-physiological interaction (PPI) analyses (GLM3)

380 to assess functional connectivity between sgACC and the whole brain as a

381 consequence of staying versus switching after no pain for other (see methods for

382 additional details of analyses). This analysis identified a significant negative

383 association between activity in sgACC and dorsolateral prefrontal cortex $(x=-46, y=38$,

$384 \mathrm{z}=26, \mathrm{k}=382, \mathrm{Z}=4.12, p=.039$, FWE-whole brain corrected after initial thresholding at $385 p<.001)$.

386

387 To understand the nature of this effect we plotted the average slope of sgACC and

388 dIPFC connectivity during stay and switch conditions (Figure $\mathbf{6 b}, \mathbf{c}$ ). This showed that 389 there was a stronger positive coupling between sgACC and dIPFC during switch 390 choices compared to stay choices after receiving no pain for another person (Figure

391 6b-c). We did not observe significant coupling between sgACC and dIPFC during 392 switch or stay choices following no pain for self (Supplemental Analyses). 
a

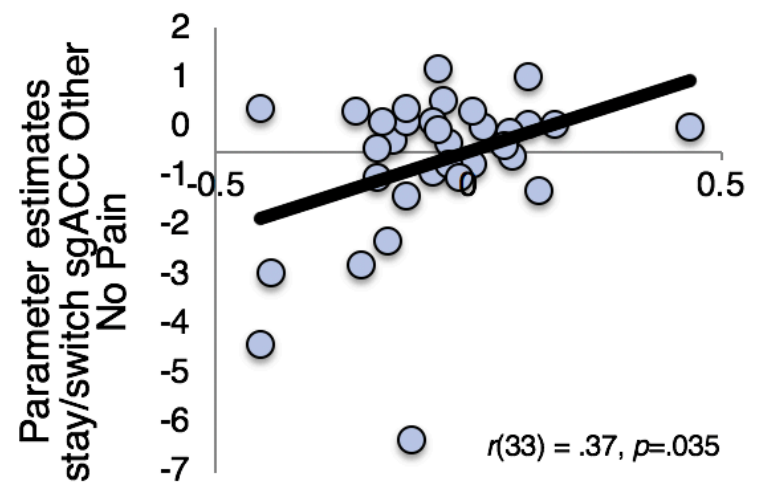

Model free $\mathrm{x}$ recipient interaction

b

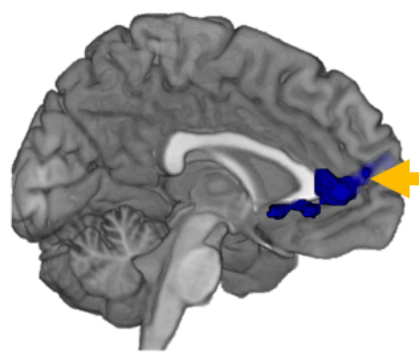

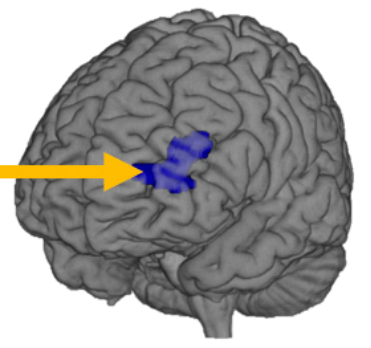

C

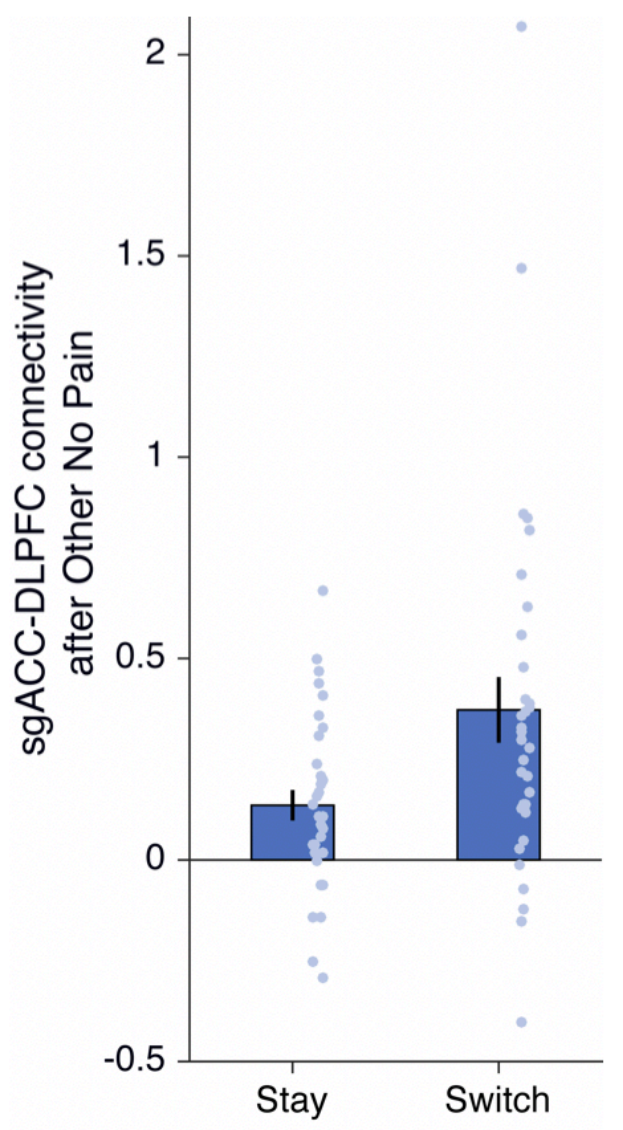

Figure 6 - Subgenual anterior cingulate cortex (sgACC) tracks stay vs. switch after no pain for other and connects more strongly to dIPFC when switching after no pain for other. (a) Bivariate association between parameter estimates for stay vs. switch after no pain for other in SgACC and greater model-free behaviour for other (more negative on $\mathrm{x}$ means relatively more model-free for other compared to self). (b) sgACC response ( $\mathrm{x}=-2, \mathrm{y}=36, \mathrm{z}=6, \mathrm{~K}=498, p=.028$, FWE whole-brain corrected after initial thresholding at $p<.001$ ) to stay vs. switch after no pain for other overlaid on the medial surface of an anatomical scan. The observed BOLD pattern is consistent with model-free behaviour. sgACC cluster connects to dorsolateral prefrontal cortex (dIPFC) $(x=-46, y=38, z=26, k=382, Z=4.12$, $p=.039$, FWE whole-brain corrected after initial thresholding at $p<.001$ ) during decisions to switch relative to stay after no pain for other. (c) Average slope estimate across participants shows stronger connectivity during switch decisions than stay decisions after receiving no pain for other between sgACC and dIPFC. Switching after no pain goes against a model-free influence.

Individual differences in moral judgment relate to model-free moral learning

410 Finally, we investigated whether individual differences in moral judgment were related 411 to individual differences in model-free moral learning and its neural basis. We

412 examined two aspects of moral judgment. First, motivated by theories suggesting that 413 'anti-utilitarian' judgments in moral dilemmas might be driven by model-free 414 processes $^{3,27}$, we measured individual differences in utilitarian reasoning using the 
415 Oxford Utilitarianism Scale ${ }^{71}$. Because our study concerned harmful outcomes, we

416 predicted that we would observe correlations with the 'instrumental harm' component

417 of the scale, which measures a permissive attitude towards harming one person in

418 order to help many others, but not the 'impartial beneficence' component of the scale,

419 which measures an impartial attitude towards helping others. First, we tested whether

420 utilitarianism was correlated with model-free behavior for others (relative to self). We

421 found a significant relationship between instrumental harm and model-free moral

422 behaviour, such that those who were the most anti-utilitarian were the most model-

423 free $(r(36)=0.37, p=0.026)$. However, there was no correlation with the impartial

424 beneficence subscale $(r(36)=-.059, p=0.731)$. Next, given past work linking

425 utilitarian reasoning with dLPFC function ${ }^{72,73}$, we predicted that utilitarianism would

426 positively predict dLPFC-sgACC connectivity when resisting model-free influence.

427 This prediction was supported, with dLPFC-sgACC connectivity positively correlated

428 with instrumental harm $(r(33)=0.43, p=0.012)$, but not impartial beneficence $(r(33)=$

$429-.11, p=0.537)$.

430

431 Second, we probed the sensitivity of moral wrongness judgments to how much

432 suffering an action inflicts on a victim ('outcome sensitivity'), versus how aversive it

433 feels to perform the action ('action sensitivity'74). Past work has connected the former

434 with model-based learning and the latter with model-free learning ${ }^{3,27}$. However, we

435 note that model-free learning is directly sensitive to recent outcomes ${ }^{18}$, which might

436 lead to an association between model-free behavior and outcome sensitivity.

437 Participants evaluated the moral wrongness of 23 harmful actions that varied

438 independently in how much suffering they would cause, versus how aversive they

439 would feel to perform. Action sensitivity and outcome sensitivity were inversely

440 correlated $(r(36)=-.40, p=.016)$. Partial correlations controlling for action sensitivity

441 revealed that outcome sensitivity was positively correlated with several aspects of

442 model-free moral learning (Figure 7), including the tendency to switch following harm

443 to others $(r(36)=-.37, p=.027)$, the strength of model-free prediction error signals for

444 other vs. self in thalamus/caudate $(r(34)=.385, p=.025)$, and the strength of model-

445 free influence in $\operatorname{sgACC}(r(33)=-.374, p=.032)$. The reverse partial correlations

446 testing for the effect of action sensitivity whilst controlling for outcome sensitivity were 

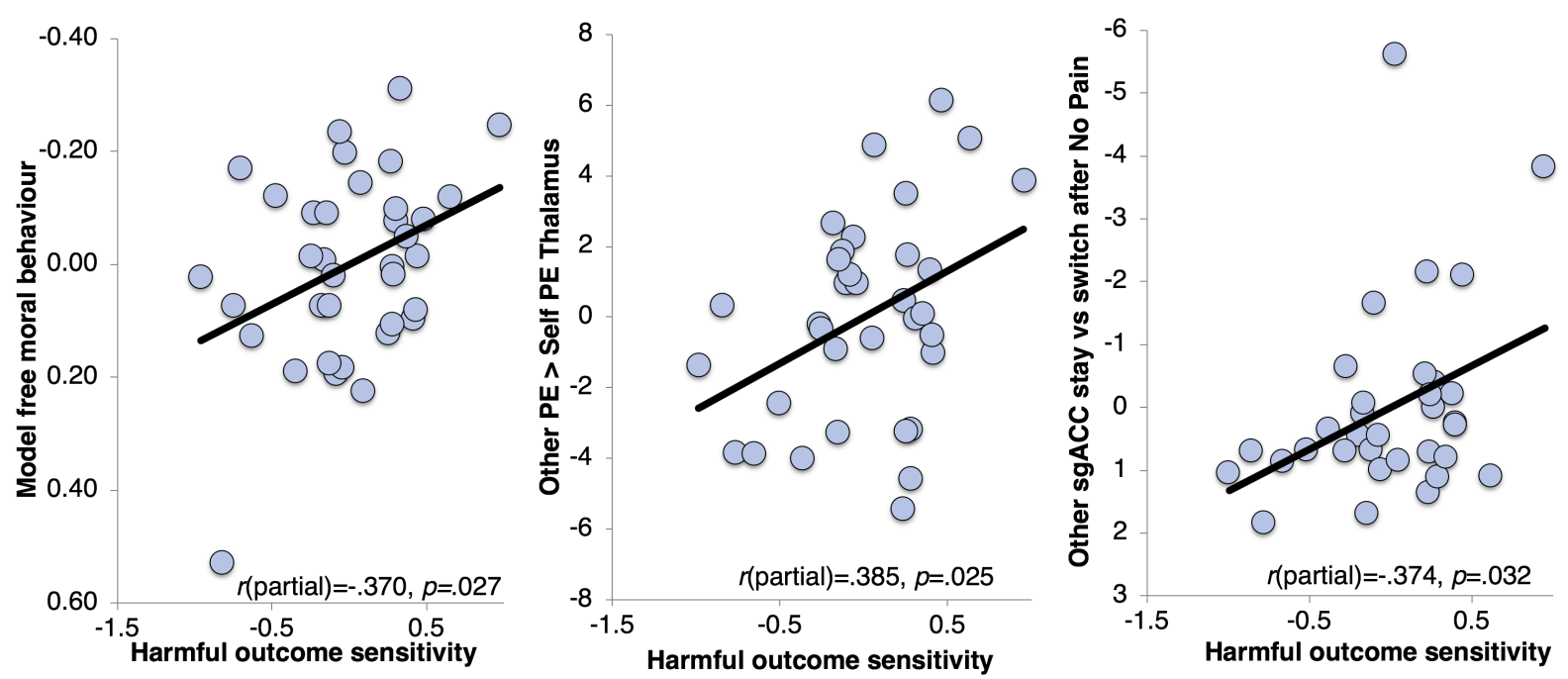

Figure 7 - Sensitivity to harm in moral judgments correlates with model-free moral behaviour and its neural correlates. (a) Partial correlation controlling for harmful action sensitivity between harmful outcome sensitivity and model-free moral behaviour. Note the values are reversed on the $y$ axis to depict that greater model-free behaviour is associated with greater harmful outcome sensitivity (b) Partial correlation controlling for harmful action sensitivity, between harmful outcome sensitivity and prediction errors of pain avoidance in the thalamus/caudate for Other compared to Self (c) Partial correlation controlling for harmful action sensitivity between harmful outcome sensitivity and parameter estimates extracted from the parametric regressor for stay vs switch after no pain for other in subgenual anterior cingulate cortex (sgACC). Note that values are reversed on the $y$ axis such that a greater tendency to stay vs switch tracked in sgACC correlates with harmful outcome sensitivity.

\section{Discussion}

Learning to avoid actions that harm other people is a fundamental prerequisite for moral behaviour. Here we show that people prioritize model-free learning when actions have the potential to harm others, and that learning to avoid harming others (vs. self) has a distinct neural signature. The thalamus/caudate differentially encoded prediction errors of pain avoidance for self versus other, whilst sgACC and right TPJ tracked positively and negatively with model-free influence on pain avoidance at the time of choice. Overriding model-free influence when choices affected others invoked stronger connectivity between sgACC and dIPFC. Finally, multiple aspects of moral judgment were associated with model-free moral learning and its neural correlates. 
476 In the context of our study, model-free moral learning manifested as a reduced

477 likelihood of repeating actions that harmed others on the previous trial, regardless of

478 whether such actions typically led to states with a high likelihood of harmful outcomes.

479 Our behavioural finding that people were more model-free when learning to avoid

480 harming others relative to themselves suggests that potentially harmful actions might

481 be prioritized for automatic avoidance as a default. Given the importance of avoiding

482 harm to others for social life, such a learning mechanism would be socially adaptive.

483 Our findings are consistent with prior work showing that harmful social choices are

484 slower than helpful choices $5,51,52$ as well as evidence that morality constrains mental

485 representations of what actions are considered possible, with harmful actions removed

486 from choice sets as a default stance ${ }^{50}$. Repeatedly assigning negative action values

487 to harmful actions over the course of one's life might automatically remove such

488 actions from consideration, even though in some cases locally harmful actions can

489 lead to wider benefits (e.g., a surgeon cutting open a patient to remove a cancerous

490 tumor). If such a learning strategy is socially adaptive, this raises interesting questions

491 about whether model-free learning can be considered an "optimal" strategy in the

492 ecological sense.

494 An alternative explanation for our behavioral findings is that model-based learning is 495 effortful26, and people choose to put in less effort to benefit others ${ }^{75}$. However, this 496 explanation seems unlikely given that model-free moral learning in our study was 497 specifically driven by a lower probability of repeating choices that harmed others. In 498 other words, participants were more likely to actively switch their choices following 499 harm to others, rather than passively sticking with the status quo. Our results were 500 also not due to differences in the subjective perception of the harmfulness or 501 aversiveness of the outcomes for others compared to self, as participants overall rated 502 shocks received for others in the task as being just as aversive as shocks received for 503 themselves. Furthermore, we did not observe differences in choice consistency 504 (captured by the temperature parameter in our model) during learning for self vs. 505 others. Since choice consistency is related to task engagement, if our behavioral 506 effects reflected reduced effort for others, we would expect to see lower choice 
507 consistency when learning for others than self. Together these alternative

508 considerations suggest that people might naturally prioritize model-free

509 strategies/behaviours when avoiding harm to others, and these effects may not simply

510 be explained by less effort or engagement when actions affect others relative to

511 oneself.

512

513 Turning to the neural findings, we observed a signal in the thalamus, extending to the

514 caudate, that differentially encoded model-free prediction errors when learning to

515 avoid harming others versus self. These subcortical regions were previously observed

516 to encode value during moral decisions to avoid profiting from others' pain 4 and play

517 a critical role in associative learning and moral-decision-making more broadly $40,42,44,45$.

518 The thalamus is often linked to the processing of the affective dimension of pain in

519 addition to its sensory properties ${ }^{76}$. For example, microstimulation of the thalamus can

520 invoke affective memories of previously experienced pain ${ }^{45}$. The thalamus/caudate

521 signal differed from the adjacent ventral striatum response that positively tracked

522 model-free prediction errors regardless of the recipient of the outcome, consistent with

523 a previous study using a similar task with rewarding outcomes for self only ${ }^{20}$. These

524 findings suggest that multiple sub-cortical areas support model-free moral learning,

525 perhaps with ventral striatum providing a generic model-free prediction error signal

526 that is insensitive to outcome valence and outcome recipient, and thalamus/caudate

527 providing additional information about social context.

529 Another signature of model-free influence was observed in sgACC at the time of

530 choice, contingent on the outcome of the previous trial and specific to the 'other'

531 condition. Specifically, signal in SgACC was higher when participants repeated actions

532 that previously avoided harming others, but not during similar choices for oneself. This

533 pattern is consistent with model-free behavior, and individual differences in model-free

534 behavior tracked with individual differences in sgACC response at the time of choice.

535 Notably, previous work has implicated sgACC in model-free learning to gain rewards

536 for others but not self 53 and in receiving unexpected positive feedback from others ${ }^{77}$,

537 suggesting this region might compute learning signals that are specific to social 
settings. More broadly, activity in sgACC has been positively associated with prosocial and moral behaviours ${ }^{15,54,55,57}$.

541 Collectively these findings suggest that sgACC might bias decision-making away from choices that could harm others. However, this default strategy is not always appropriate, for instance in settings where a typically harmful action might lead to a better outcome. We observed signals at the time of choice in two areas that negatively tracked with model-free influence. The first, in right TPJ, showed a response pattern precisely opposite to that observed in SgACC. Signal in rTPJ was higher when participants abandoned a choice that previously avoided pain for others, but not during similar choices for oneself. In addition, overriding model-free influence at the time of choice was associated with increased functional connectivity between SgACC and dLPFC. These two regions showed stronger coupling on trials where participants abandoned a choice that previously avoided pain for others, compared with trials where participants repeated actions that previously spared others from pain. Although we cannot confidently attribute these patterns to model-based control, past work has implicated dLPFC and TPJ in model-based learning and decision-making ${ }^{31,70 . ~ O n e ~}$ intriguing possibility is that SgACC promotes social harm avoidance as a default, while TPJ and dLPFC provide contextual information that enables this default to be overridden when appropriate. Research implicating these regions in the adjustment of moral decisions to blame and punishment provides initial support for this possibility $5,32,33,39,47$.

Finally, we observed correspondences between model-free learning, its neural substrates, and moral judgments. Theoretical work has proposed links between model-based/model-free learning and moral judgment $3,27,28,78$, but empirical support for such links has been scarce. We probed two aspects of moral judgment. First, we examined individual differences in the dimension of 'utilitarian' moral reasoning that justifies harming one person to help many others. Consistent with our predictions, as well as work highlighting a link between dLPFC activity and 'utilitarian' judgments' we found a positive relationship between instrumental harm and dLPFC-sgACC 
connectivity when resisting model-free influence. We also observed that those who were the most anti-utilitarian were the most model-free.

572 Second, using a task that asks participants to judge how morally wrong it would be to perform a series of violent actions that varied independently in terms of how much suffering they would inflict ('outcome sensitivity'), versus how aversive they felt to perform ('action sensitivity') ${ }^{74}$. We found that individual variability in both the behavioural and neural signatures of model-free learning was specifically correlated with outcome sensitivity, but not action sensitivity. Those people whose moral wrongness judgments were more sensitive to the severity of harmful outcomes were less likely to repeat decisions that harmed others, and showed stronger model-free prediction error signals in the thalamus and caudate, and stronger responses in sgACC when repeating decisions that previously avoided harming others. Model-free learning has previously been suggested to explain why actions that typically harm others feel aversive to perform, even when they are not actually harmful3,27. Thus, one might expect that a greater tendency to engage in model-free moral learning should predict action sensitivity in moral judgments, rather than outcome sensitivity. However, model-free learning is directly sensitive to recent outcomes ${ }^{18}$, and in the context of our task, manifested as a tendency for choices to be immediately sensitive to harmful outcomes for others. Thus, individual differences in sensitivity to others' harm could be commonly associated with model-free moral learning and outcome sensitivity in moral judgments. Overall, these findings provide preliminary evidence linking modelfree learning to individual differences in moral judgments, effects that could be investigated more extensively in larger samples.

594 More broadly, our findings highlight differences in the neurocognitive mechanisms engaged in learning to avoid harming oneself versus others that underscore the unique demands of social decision-making. One important feature of decisions that affect others (as opposed to oneself) is that it is far more difficult to build a model that incorporates others' preferences and beliefs than a model that captures only one's own preferences. Furthermore, it is impossible to evaluate the accuracy of such models, given that the subjective experiences of others are fundamentally 
601 unknowable ${ }^{79,80}$. Utilitarian approaches to moral decision-making that involve

602 maximizing well-being for all sentient beings ${ }^{81}$ may thus be computationally intractable

603 for the model-based system. Rule-based approaches, like those enshrined in

604 deontological theories of morality ${ }^{82}$ circumvent the need for complex model building

605 and may be socially adaptive even for simple social decisions like the ones studied

606 here. Whether the prioritization of model-free learning extends to other kinds of social

607 decisions, such as acting to obtain rewards for others, avoiding monetary losses or 608 indeed even social decision-making in nonhuman species, is an important topic for

609 future study.

610

611 Overall, we observed that when learning to avoid harm to others (versus self),

612 participants showed a stronger relative balance toward model-free over model-based

613 learning. Multiple model-free learning signatures were apparent in behaviour as well

614 as cortical and subcortical areas that distinctly process harm avoidance for others

615 compared to self. These findings could have important implications for theories of

616 learning and moral-decision-making as well as disorders associated with impaired

617 avoidance learning and social cognition.

618

619 Methods

620

\section{Experimental procedures}

\section{Participants}

623 Forty-one right-handed healthy adults were recruited through university participant

624 databases. Exclusion criteria included previous or current neurological or psychiatric

625 disorder, non-normal or non-corrected to normal vision, previous participation in

626 studies involving social interactions and/or electric shocks and contraindications that

627 prohibited MRI scanning. One participant was excluded as they reported that they did

628 not believe their decisions would affect another person in the post-scanning debrief.

629 Three participants were excluded because the logistic regression analysis of their

630 choice behaviour did not converge. In two of these participants, this was because they

631 had less than $5 \%$ switch trials; in the third participant, there was a very high correlation 
632 (>0.8) between the outcome and transition regressors on switch trials. One participant

633 was excluded from the fMRI analysis due to distortions in the scan caused by metal 634 artefacts (braces) and another for excessive head motion. This left a final sample of 63536 participants for behavioural analyses (16 female, age 18-36) and 34 participants

636 (16 female, age 18-36) for the parametric fMRI analyses. For the stay/switch analysis 637 one further participant was excluded for having no variance in at least one regressor 638 making their fMRI GLM inestimable. With 33 subjects we had $80 \%$ power to detect a 639 'medium' effect size of $d=0.50$ at alpha $=0.05$ (two-tailed), an effect size smaller than 640 typically reported in this field, indicating sufficient power. All participants gave written 641 informed consent and the study was approved by the University of Oxford Medical 642 Sciences Division Ethics Committee.

\section{Procedure}

646 Participants completed a pain thresholding procedure which was based on previous 647 studies of self and other pain processing ${ }^{4,5}$. The pain thresholding procedure allowed 648 us to control for heterogeneity of skin resistance between participants to ensure the delivered shocks would be rated at a matched subjective level of pain intensity and also to provide participants with full experience of the shocks before the learning task to ensure their choices were truly guided by knowledge of the pain and no pain outcomes. Participants were then assigned to roles of either 'decider' or 'receiver' using a role assignment procedure that has been used in several previous studies $\left(s^{5} e^{5,75}\right)$. Briefly, participants were instructed to wear coloured rubber gloves to hide their identity. They then stood either side of a door and waved to one another so that they knew another person was there but could not discern any information about the other participant's age or gender. Next a coin was flipped to decide who would draw a ball out of a box first and then each participant drew a ball. The experimental participant was then told that the colour of their ball meant they had been assigned to

660 the role of decider. Before completing the task in the scanner participants performed 661 a practice task of one block that did not specify whether outcomes were for self or 662 other and they were told no actual shocks would be received during the practice. This 663 practice task, which used stimuli not used in the experimental task, allowed 
participants to become familiar with the transition structure of the task, that they were told would remain the same in the main experiment. The main experiment consisted of 4 blocks of 68 trials (136 trials for self and 136 trials for other) and lasted $\sim 45$ minutes.

\section{Experimental task}

We adapted features from two variants of a task designed to distinguish model-free and model-based learning 20,26 (Figure 1, Supplemental Experimental note). Participants were presented with two fractal images that probabilistically led them to one of two 'states' where they were required to make a second choice that was followed by a symbol indicating the receipt of pain or the receipt of no pain (neutral). At the beginning of each block and on each trial, they were told whether they were playing for themselves ('You') meaning the painful outcomes would be delivered to themselves, or for the other participant ('Receiver'), indicating that the painful outcomes would be delivered to the other participant. In order to match the self and other conditions in terms of pain stimulation, no electric shocks were delivered during the scan. However, participants were told that $10 \%$ of the electric shocks that they acquired during the task would be given to themselves at the end of the session and $10 \%$ of the electric shocks they accumulated for the other participant would be delivered to the other participant at the end of the scanning session. In order to account for potential differences in pain perception, participants were instructed that for 'You' trials we would use the voltage setting that corresponded to their level 8 rating, and for the "Receiver" trials, we would use the voltage setting that corresponded to the Receiver's level 8 rating (full instructions can be downloaded at (OSF https://osf.io/3stp9/files/). At the end of the scan participants also rated how they felt when obtaining a shock for themselves and the receiver during that task on a 0-10 point scale from 'very negative' (0) to 'very positive' (10). Participants perceived the shocks to be aversive for both self $(M=3.38, S D=1.44)$ and other $(M=3.30, S D=1.61)$ $(t(36)=.279, p=.782)$ suggesting that the perception of harm was equivalent.

693 Participants were instructed that the probability of reaching each of the two 'states' 
695

696

697

698

699

700

701

702

703

704

705

706

707

708

709

710

711

712

713

714

715

716

717

718

719

720

721

722

723

724

725

deliver pain or no pain would change throughout the task so that they needed to keep on learning. Adding the 'receiver' trials meant that fewer trials, overall, were included in each condition compared to the original paradigm ${ }^{20}$. We therefore made two modifications to the original paradigm in order to better sample variability in behaviour. We bounded the probability that the four second-stage options would deliver pain between 0 and 1 (instead of 0.25 and $0.75 \mathrm{in}^{20}$ ) and we increased the drift rate of the probabilities to 0.2 (instead of $0.025 \mathrm{in}^{20}$ ). Moreover, we specifically chose to implement the main aspects off the Daw et al. ${ }^{20}$, version of the paradigm rather than using all the modifications described $i^{26}$, as the Daw et al. ${ }^{20}$, paradigm allowed us to assess peoples' relative balance between model-free or model-based behaviour 20,26 when there was no particular incentive to being more model-free or model-based on the task because the degree of model-basedness did not affect the number of shocks received.

\section{Statistical analysis of behavioral data}

Analyses of behavioural data were performed in SPSS 25 (Armonk, New York: IBM Corp, for bivariate correlations), R (version 1.1.423, for linear mixed-effects modelling, Ime4, version 1.1-21) and Matlab 2015b (for logistic regression analyses on the probability of stay). For Fig $2 a$, we calculated the $\%$ of stay choices after common or rare transitions following pain or no pain outcomes $(2 \times 2)$. For regression analyses using Ime4 in R, we coded Stay as 1 and Switch as 0 and created regressors for transition type, outcome, outcome $x$ transition type, agent $x$ outcome and agent $x$ outcome $\mathrm{x}$ transition type. We used Bound Optimization by Quadratic Approximation (bobyqa) with $1 \mathrm{e} 5$ function evaluations. We examined bivariate associations between the interactions with agent from the regression analyses and neural responses with individual differences in utilitarianism using the Oxford Utilitarianism Scale (OUS) ${ }^{71}$ and action and outcome sensitivity from the Harmful Action Outcome Scale ${ }^{74}$.

\section{Moral judgment measures}

Participants completed the OUS instrumental harm (OUS-IH), OUS Impartial Beneficence (OUS-IB) scales and the HAO scales via an online link in the preceding weeks before the scanning session. The OUS-IH consists of 4 items reflecting a 
relative willingness to cause harm to others in order to bring about the greater good

727 (e.g., "It is morally right to harm an innocent person if harming them is a necessary 728 means to helping several other innocent people"). The OUS-IB subscale consists of 5 729 items reflecting endorsement of the impartial maximization of the greater good even 730 at a cost to oneself (e.g. if the only way to save another person's life during an 731 emergency is to sacrifice one's own leg, then one is morally required to make this sacrifice). Participants rated these items on a 7-point scale $(1=$ strongly disagree, $7=$ strongly agree). A mean score was then computed for all participants.

For the HAO participants were presented with a scenario about two people, Carl and John. They are told John is terminally ill and sincerely wants to die and has asked another person, Carl, to perform a mercy killing. Participants then rated how morally wrong each of the 23 methods of killing were on a scale from 1 - the least morally wrong, to 10 - the most morally wrong. In a previous study ${ }^{74}$ participants were asked to rate the action value and outcome value of these different methods of killing. To assess action value, the researchers asked participants to rate how upsetting it would be to "act out" performing each behavior as though it were part of a movie script. To assess outcome value, they asked them to rate how much suffering each act would impose. We used the mean action and outcome scores derived from this initial paper to predict the 'wrongness' scores in our current sample. This analysis created two different beta weights for each participant corresponding to the action and outcome sensitivity, respectively.

\section{Computational modelling of behavioural data}

751 For modelling of choice behaviour using trial-by-trial updates, we proceeded in two steps. First, we evaluated a number of models separately based on their performance on the self and other blocks. This was to probe whether the same model would win for self and other blocks, allowing us to rule out participants might employ entirely different strategies in the two block types. The following models were fitted: 
(1) 7-parameter: full model specified by Daw et al. using parameters: learning rates for stage 1 and $2(\alpha S 1, \alpha S 2)$, temperature parameters for stage 1 and 2 ( $\beta S 1, \beta S 2)$, a perseverance parameter $(\rho)$, an eligibility trace $(\lambda)$ and a model-free/based weighting parameter $(\omega)$; for full details of model see Supplementary information and 20

(2) 6-parameter model, as (1) but with $\lambda=1$ ( $\lambda$ was shown to have a high mean value and small variance in previous work e.g. ${ }^{62}$ )

763

(3) 5-parameter model, as (1) but with only one $\alpha$ and $\beta$ for stage 1 and 2

(4) 4-parameter model, as (3) but with $\lambda=1$

(5) 5-parameter model, as (4) but with two learning rates for pain and no pain outcomes ( $\alpha$ Pain, $\alpha$ NoPain)

Models were fitted using a hierarchical Bayesian model fitting approach described in detail in 83,84 . It finds the maximum a posteriori estimate of each parameter for each subject using a prior distribution for each parameter which helps to regularise and constrain parameters. The algorithm uses Expectation-Maximization (EM) ${ }^{85}$ and parameters were transformed to a logistic or exponential distribution to enforce constraints and ensure normality such that $0<\{\alpha, \omega\}<1,\{\beta, \lambda\}>0$.

For formal model comparison, we report the Bayesian Information Criterion (BIC) based on the log-likelihood, and computed the model evidence by integrating out the free parameters (BICint ${ }^{83,84}$; Supplementary Tables 2 and 4). Exceedance probabilities were calculated by feeding the BICint into SPM's function spm_BMS (http://www.fil.ion.ucl.ac.uk/spm/software/spm8).

The five-parameter model with separate learning rates for pain and no-pain outcomes best explained behaviour in both self and other conditions (Supplementary Table 2). We report the difference between the best-fitting parameters, but this method has a caveat. Because of the nature of hierarchical fitting, which uses separate priors for self and other parameters, this method is somewhat biased towards finding differences. Meanwhile, fitting self and other parameters using the same priors, is overly conservative and biased against finding differences. 
To resolve whether the parameter $\omega$ differed between self and other blocks, in line with results from the basic logistic regression analyses, and without introducing any such biases, in a second step, we therefore fitted three models to the merged data of both self and other blocks:

(1) 5-parameter model (as (5) above) with all parameters shared between self/other

(2) 6-parameter model with $\alpha$ Pain, $\alpha$ NoPain, $\beta$ and $\rho$ shared but $\omega$ split into $\omega$ Self and $\omega$ Other

(3) 7-parameter model with $\alpha$ Pain, $\alpha$ NoPain, $\beta$ shared and $\rho$ and $\omega$ split into $\rho$ Self, $\rho$ Other, $\omega$ Self and $\omega$ Other

As described above, model comparison was performed based on BICint values

(Supplementary Table 4). The mean parameter estimates are shown in

Supplementary Fig 3 and Supplementary Table 5. We also simulated data from our

\section{$804 \quad$ FMRI acquisition and analysis}

805 Multiband T2*-weighted echo planar imaging (EPI) volumes with blood oxygenation806 level-dependent (BOLD) contrast were acquired using a Siemens Prisma 3T MRI 807 scanner. The EPI volumes were acquired in an ascending manner, at an oblique angle $808\left(\approx 30^{\circ}\right)$ to the AC-PC line to decrease the impact of susceptibility artefacts in the 809 orbitofrontal cortex. We used the following parameters. Voxel size $2 \times 2 \times 2$, TE=30 $810 \mathrm{~ms}$; repetition time $=1570 \mathrm{~ms}$; flip angle $=90^{\circ}$; field of view $=216 \mathrm{~mm}$. The structural scan 811 was acquired using a magnetization prepared rapid gradient echo (MPRAGE) 812 sequence with 192 slices; slice thickness=1 mm; TR=1900 ms; TE=3.97 ms; field of

813 view=192 mm x 192mm; voxel size=1×1×1 mm resolution.

815 Fmri data were analysed using SPM12 (www.fil.ion.ucl.ac.uk/spm). Images were 816 realigned and unwarped using a fieldmap and co-registered to the participant's own 817 anatomical image. The anatomical image was processed using a unified segmentation 818 procedure combining segmentation, bias correction, and spatial normalization to the 819 MNI template using the New Segment procedure; the same normalization parameters 
820 were then used to normalize the EPI images. Lastly, a Gaussian kernel of $8 \mathrm{~mm}$

821 FWHM (SPM default) was applied to spatially smooth the images.

823 Before the study, example first-level design matrices were checked to ensure that 824 estimable GLMs could be performed with independence between the parametric 825 regressors: value difference at the first-stage choice, the state prediction error at stage 8262 , and a model-free prediction error at the time of the outcome. This allowed us to look 827 at value and prediction error responses independent from one another. We also tested 828 a GLM that coded switch vs. stay trials as a parametric modulator at the time of choice 829 dependent on the previous outcome. Again this GLM could be estimated with 830 independence (See Supplementary Figure 1). We convolved these different event 831 types with SPM's canonical haemodynamic response function. All events were 832 modelled as stick functions with 0 duration.

834 For GLM1, each of these regressors was associated with parametric modulators

835 taken from the computational model. At the time of the first stage choice this was the 836 value difference from the hybrid model combining model-free and model based

837 learning. At the time of the second stage choice this was the state prediction error for 838 the transition from stage 1 to stage 2, and at the time of the outcome this was the 839 model free prediction error (since the behavioural differences were in the model-free 840 parameters). In all cases, values were modelled separately for the onsets of self and 841 other trials. As in ${ }^{20}$, we fixed the parameters to the average values for self and other

842 (Supplementary Table 4) but allowed $\omega$ to vary.

844 For GLM2 we modelled whether participants stayed or switched at the first-stage choice relative to the outcome on the previous trial, i.e. no pain or pain. Due to the smaller number of trials included in this analysis we coded stay and switch as a parametric regressor with values of 1 assigned to switch and -1 assigned to stay. One participant did not have a trial in at least one of these regressors and was therefore excluded from the stay-switch analysis. For all GLMs in some participants, an extra regressor modelled all missed trials, on which participants did not select one of the first-stage choices. 
853 GLM3 and GLM4 corresponded to our psychophysiological interaction analyses. We

854 defined a seed region in the sgACC using a $6 \mathrm{~mm}$ sphere based on the peak co855 ordinates from our analyses (for completeness we also ran PPI analyses using seed regions in thalamus and TPJ, see Supplementary Text). We then extracted the physiological variable and the psychophysiological interaction terms for stay vs. switch after no pain for other (GLM3) and stay vs. switch after no pain for self as a control analysis (GLM4). These PPI terms were entered into the GLMs along with all previous regressors that specified the events of our study as described above. In all GLMs, six head motion parameters modelled the residual effects of head motion as covariates of no interest. Data were high-pass filtered at $128 \mathrm{~s}$ to remove low-frequency drifts, and the statistical model included an $A R(1)$ autoregressive function to account for autocorrelations intrinsic to the fMRI time-series.

Contrast images from the first level were input into flexible-factorial designs. Following standard procedures, main effects are reported at $p<.05$, family-wise error (FWE) cluster corrected across the whole brain after initial thresholding at $p<.001$, or $p<0.05$ FWE small volume corrected (SVC) after initial thresholding at $p<.001$ for regions where we had a strong a priori hypothesis ${ }^{87,88}$. These areas were defined anatomically and included subcortical regions of the caudate and thalamus (taken from the WFU PickAtlas Toolbox), bilateral posterior TPJ (from ${ }^{89}$ ) the sgACC (areas s24 and 25 from $^{90}$ and the dIPFC (areas $46 \mathrm{v}$ and 9 taken from ${ }^{91}$ ). These ROls were also used to confirm anatomical labelling. We additionally applied a false discovery rate correction (FDR) for the number of ROI corrections. All ROI comparisons remained significant $(p<0.05)$ when controlling for the number of comparisons using FDR.

\section{Author contributions}

880 P.L.L, M. K-F and M. J. C designed study. P.L.L and A. A. collected data. P.L.L and

\section{Acknowledgements}


885 This work was supported by a Medical Research Council Fellowship (MR/P014097/1),

886 a Christ Church Junior Research Fellowship and a Christ Church Research Centre 887 Grant to P. L. L., a Wellcome Trust grant (106164/A/14/Z) and an Academy of Medical 888 Sciences (SBF001\1008) grant to MJC. MCKF was supported by a Sir Henry 889 Wellcome Fellowship (103184/Z/13/Z). The Wellcome Centre for Integrative 890 Neuroimaging is supported by core funding from the Wellcome Trust (203139/Z/16/Z).

891 We would like to thank Ms. Eloise Copland and Dr. Hongbo Yu for assistance with 892 data collection, Dr. Marco Wittman, Dr. Peter Smittenaar, Dr. Quentin Huys, Dr. Elsa 893 Fouragnan, and Dr. Mehdi Keramati for assistance with data analysis, and Dr. 894 Matthew Apps, Prof. Nathaniel Daw, Prof Peter Dayan, Dr Wouter Kool and Ms Mary 895 Montgomery for helpful discussions.

896

\section{Declaration of interests}

898

899 The authors have no competing interests

900

901

Materials and correspondence

902

903 Please address correspondence to Patricia L. Lockwood, Miriam C Klein-Flugge, and 904 Molly J Crockett.

905

906

\section{Data availability}

907

908 All data and code used to generate the figures can be downloaded at: [OSF

909 https://osf.io/3stp9/files/]

910

911 Unthresholded statistical maps can be downloaded at: [Identifier to be added upon

912 publication] 
1. Gert, B. Common Morality: Deciding What to Do. (Oxford University Press,

918 2004).

2. Gray, K., Young, L. \& Waytz, A. Mind Perception Is the Essence of Morality. Psychol. Inq. 23, 101-124 (2012).

3. Crockett, M. J. Models of morality. Trends Cogn. Sci. 4-7 (2013). doi:10.1016/j.tics.2013.06.005

4. Crockett, M. J., Siegel, J. Z., Kurth-Nelson, Z., Dayan, P. \& Dolan, R. J. Moral transgressions corrupt neural representations of value. Nat. Neurosci. 20, 879885 (2017).

926

5. Crockett, M. J., Kurth-nelson, Z., Siegel, J. Z., Dayan, P. \& Dolan, R. J. Harm to others outweighs harm to self in moral decision making. Proc. Natl. Acad. Sci. 112, 201424572 (2015).

6. Cushman, F., Kumar, V. \& Railton, P. Moral learning: Psychological and philosophical perspectives. Moral Learn. 167, 1-10 (2017).

7. Decety, J. \& Cowell, J. M. Interpersonal harm aversion as a necessary foundation for morality: A developmental neuroscience perspective. Dev. Psychopathol. 30, 153-164 (2018).

8. Cushman, F., Gray, K., Gaffey, A. \& Mendes, W. B. Simulating murder: The aversion to harmful action. Emotion 12, 2 (2012).

936 9. Blair, R. J. R. The neurobiology of psychopathic traits in youths. Nat. Rev. Neurosci. 786-799 (2013).

938 10. Kohlberg, L. Stages in the development of moral thought and action. (1969). 
11. Tremblay, R. E. The development of agressive behaviour during childhood: What have we learned in the past century? Int. J. Behav. Dev. 24, 129-141 (2000).

12. Henrich, J., Heine, S. J. \& Norenzayan, A. The weirdest people in the world? Behav. Brain Sci. 33, 61-83 (2010).

13. Gino, F., Ayal, S. \& Ariely, D. Contagion and differentiation in unethical behavior: The effect of one bad apple on the barrel. Psychol. Sci. 20, 393-398 (2009).

14. Nolan, J. M., Schultz, P. W., Cialdini, R. B., Goldstein, N. J. \& Griskevicius, V. Normative social influence is underdetected. Pers. Soc. Psychol. Bull. 34, 913-

15. Wittmann, M. K., Lockwood, P. L. \& Rushworth, M. F. S. Neural Mechanisms of Social Cognition in Primates. Annu. Rev. Neurosci. (2018). doi:10.1146/annurevneuro-080317-061450

16. Schultz, W. Updating dopamine reward signals. Curr. Opin. Neurobiol. 23, 229238 (2013).

17. Dayan, P. \& Daw, N. D. Decision theory, reinforcement learning, and the brain. Cogn. Affect. Behav. Neurosci. 8, 429-453 (2008).

18. Sutton, R. S. \& Barto, A. G. Reinforcement learning: an introduction. (MIT press, 1998).

19. O'doherty, J. P., Cockburn, J. \& Pauli, W. M. Learning, reward, and decision making. Annu. Rev. Psychol. 68, 73-100 (2017).

20. Daw, N. D., Gershman, S. J., Seymour, B., Dayan, P. \& Dolan, R. J. Modelbased influences on humans' choices and striatal prediction errors. Neuron 69, 1204-1215 (2011). 
21. Glascher, J., Daw, N., Dayan, P. \& O’Doherty, J. P. States versus Rewards: Free Reinforcement Learning. Neuron 66, 585-595 (2010).

22. Lee, S. W., Shimojo, S. \& O’Doherty, J. P. Neural computations underlying arbitration between model-based and model-free learning. Neuron 81, 687-699 (2014).

23. Wunderlich, K., Dayan, P. \& Dolan, R. J. Mapping value based planning and extensively trained choice in the human brain. Nat. Neurosci. 15, 786 (2012).

24. Tricomi, E., Balleine, B. W. \& O'Doherty, J. P. A specific role for posterior dorsolateral striatum in human habit learning. Eur. J. Neurosci. 29, 2225-2232 (2009).

25. Daw, N. D., Niv, Y. \& Dayan, P. Uncertainty-based competition between prefrontal and dorsolateral striatal systems for behavioral control. Nat. Neurosci. 8, 1704-11 (2005).

26. Kool, W., Cushman, F. A. \& Gershman, S. J. When does model-based control pay off? PLoS Comput. Biol. 12, e1005090 (2016).

27. Cushman, F. Action, outcome, and value: A dual-system framework for morality. Personal. Soc. Psychol. Rev. 17, 273-292 (2013).

28. Gęsiarz, F. \& Crockett, M. J. Goal-directed, habitual and Pavlovian prosocial behavior. Front. Behav. Neurosci. 9, 135 (2015).

982 29. Greene, J. D. The rat-a-gorical imperative: Moral intuition and the limits of affective learning. Moral Learn. 167, 66-77 (2017). judgments? Cognition 150, 232-242 (2016). 
31. Smittenaar, P., FitzGerald, T. H., Romei, V., Wright, N. D. \& Dolan, R. J. model-free control in humans. Neuron 80, 914-919 (2013).

32. Carlson, R. W. \& Crockett, M. J. The lateral prefrontal cortex and moral goal pursuit. Curr. Opin. Psychol. (2018).

991

33. Spitzer, M., Fischbacher, U., Herrnberger, B., Gron, G. \& Fehr, E. The neural signature of social norm compliance. Neuron 56, 185-196 (2007).

34. Greene, J. D. \& Paxton, J. M. Patterns of neural activity associated with honest and dishonest moral decisions. Proc. Natl. Acad. Sci. 106, 12506-12511 (2009).

35. Buckholtz, J. W. \& Marois, R. The roots of modern justice: cognitive and neural foundations of social norms and their enforcement. Nat. Neurosci. 15, 655 (2012).

36. Chang, L. J., Smith, A., Dufwenberg, M. \& Sanfey, A. G. Triangulating the neural, psychological, and economic bases of guilt aversion. Neuron 70, 560-572 (2011).

37. Feldmanhall, O. et al. Differential Neural Circuitry and Self-Interest in Real versus Hypothetical Moral Decisions. Soc. Cogn. Affect. Neurosci. 1-23 (2012). doi:10.1093/scan/nss069

38. Knoch, D., Pascual-Leone, A., Meyer, K., Treyer, V. \& Fehr, E. Diminishing reciprocal fairness by disrupting the right prefrontal cortex. science 314, 829-832 (2006).

39. Ruff, C. C., Ugazio, G. \& Fehr, E. Changing social norm compliance with noninvasive brain stimulation. Science 342, 482-484 (2013). 
1009

1010

1011

1012

40. Fumagalli, M. \& Priori, A. Functional and clinical neuroanatomy of morality. Brain 135, 2006-2021 (2012).

41. Gaffan, D. \& Murray, E. A. Amygdalar interaction with the mediodorsal nucleus of the thalamus and the ventromedial prefrontal cortex in stimulus-reward associative learning in the monkey. J. Neurosci. 10, 3479-3493 (1990).

42. Kim, H., Shimojo, S. \& O’Doherty, J. P. Is Avoiding an Aversive Outcome Rewarding? Neural Substrates of Avoidance Learning in the Human Brain. PLOS Biol. 4, e233 (2006).

43. Oyoshi, T., Nishijo, H., Asakura, T., Takamura, Y. \& Ono, T. Emotional and Behavioral Correlates of Mediodorsal Thalamic Neurons during Associative Learning in Rats. J. Neurosci. 16, 5812-5829 (1996).

44. Treede, R.-D., Kenshalo, D. R., Gracely, R. H. \& Jones, A. K. The cortical representation of pain. Pain 79, 105-111 (1999).

45. Lenz, F. A. et al. Stimulation in the human somatosensory thalamus can reproduce both the affective and sensory dimensions of previously experienced pain. Nat. Med. 1, 910-913 (1995).

46. FeldmanHall, O., Mobbs, D. \& Dalgleish, T. Deconstructing the brain's moral network: dissociable functionality between the temporoparietal junction and ventro-medial prefrontal cortex. Soc. Cogn. Affect. Neurosci. 9, 297-306 (2014).

47. Young, L., Camprodon, J. A., Hauser, M., Pascual-Leone, A. \& Saxe, R. Disruption of the right temporoparietal junction with transcranial magnetic stimulation reduces the role of beliefs in moral judgments. Proc. Natl. Acad. Sci. U. S. A. 107, 6753-6758 (2010). 
1032

1033

1034

1035

1036

1037

1038

1039

1040

1041

1042

1043

1044

1045

1046

1047

1048

1049

1050

1051

1052

1053

1054

48. Van Bavel, J. J., FeldmanHall, O. \& Mende-Siedlecki, P. The neuroscience of moral cognition: from dual processes to dynamic systems. Curr. Opin. Psychol.

$$
\text { 6, 167-172 (2015). }
$$

49. Öhman, A., Eriksson, A. \& Olofsson, C. One-trial learning and superior resistance to extinction of autonomic responses conditioned to potentially phobic stimuli. J. Comp. Physiol. Psychol. 88, 619 (1975).

50. Phillips, J. \& Cushman, F. Morality constrains the default representation of what is possible. Proc. Natl. Acad. Sci. 114, 4649-4654 (2017).

51. Crockett, M. J. et al. Dissociable Effects of Serotonin and Dopamine on the Valuation of Harm in Moral Decision Making. Curr. Biol. 25, 1852-1859 (2015).

52. Zaki, J. \& Mitchell, J. P. Intuitive prosociality. Curr. Dir. Psychol. Sci. 22, 466-470 (2013).

53. Lockwood, P. L., Apps, M. A. J., Valton, V., Viding, E. \& Roiser, J. P. Neurocomputational mechanisms of prosocial learning and links to empathy. Proc. Natl. Acad. Sci. 113, 9763-9768 (2016).

54. Lockwood, P. L. \& Wittmann, M. K. Ventral anterior cingulate cortex and social decision-making. Neurosci. Biobehav. Rev. 92, 187-191 (2018).

55. Cutler, J. \& Campbell-Meiklejohn, D. A comparative fMRI meta-analysis of altruistic and strategic decisions to give. Neurolmage 184, 227-241 (2019).

56. FeldmanHall, O., Dalgleish, T., Evans, D. \& Mobbs, D. Empathic concern drives costly altruism. Neuroimage 105, 347-356 (2015).

57. Moll, J., Zahn, R., de Oliveira-Souza, R., Krueger, F. \& Grafman, J. The neural basis of human moral cognition. Nat. Rev. Neurosci. 6, 799 (2005). 
1055

1056

1057

1058

1059

1060

1061

1062

1063

1064

1065

1066

1067

1068

1069

1070

1071

1072

1073

1074

1075

1076

1077

58. Moll, J. \& Schulkin, J. Social attachment and aversion in human moral cognition. Neurosci. Biobehav. Rev. 33, 456-465 (2009).

59. Wunderlich, K., Smittenaar, P. \& Dolan, R. J. Dopamine enhances model-based over model-free choice behavior. Neuron 75, 418-424 (2012).

60. Seymour, B. Pain: A Precision Signal for Reinforcement Learning and Control. Neuron 101, 1029-1041 (2019).

61. Wang, O., Lee, S. W., O’Doherty, J., Seymour, B. \& Yoshida, W. Model-based and model-free pain avoidance learning. Brain Neurosci. Adv. 2, 2398212818772964 (2018).

62. Otto, A. R., Raio, C. M., Chiang, A., Phelps, E. A. \& Daw, N. D. Working-memory capacity protects model-based learning from stress. Proc. Natl. Acad. Sci. 110, $20941-20946$ (2013).

63. Gillan, C. M., Kosinski, M., Whelan, R., Phelps, E. A. \& Daw, N. D. Characterizing a psychiatric symptom dimension related to deficits in goaldirected control. Elife 5, e11305 (2016).

64. Costa, V. D., Monte, O. D., Lucas, D. R., Murray, E. A. \& Averbeck, B. B. Amygdala and ventral striatum make distinct contributions to reinforcement learning. Neuron 92, 505-517 (2016).

65. Eldar, E., Hauser, T. U., Dayan, P. \& Dolan, R. J. Striatal structure and function predict individual biases in learning to avoid pain. Proc. Natl. Acad. Sci. 113, $4812-4817$ (2016).

66. Lockwood, P. L. et al. Neural mechanisms for learning self and other ownership. Nat. Commun. 9, (2018). 
1078

1079

1080

1081

1082

1083

1084

1085

1086

1087

1088

1089

1090

1091

1092

1093

1094

1095

1096

1097

1098

1099

1100

1101

67. Chong, T. T.-J. et al. Neurocomputational Mechanisms Underlying Valuation of Effort Costs. Plos Biol. (in press).

68. Klein-Flügge, M. C., Kennerley, S. W., Friston, K. \& Bestmann, S. Neural Signatures of Value Comparison in Human Cingulate Cortex during Decisions Requiring an Effort-Reward Trade-off. J. Neurosci. 36, 10002-10015 (2016).

69. Tobler, P. N., O’Doherty, J. P., Dolan, R. J. \& Schultz, W. Human neural learning depends on reward prediction errors in the blocking paradigm. J. Neurophysiol. 95, 301-310 (2006).

70. Dolan, R. J. \& Dayan, P. Goals and habits in the brain. Neuron 80, 312-325 (2013).

71. Kahane, G. et al. Beyond sacrificial harm: A two-dimensional model of utilitarian psychology. (2017).

72. Greene, J. D., Nystrom, L. E., Engell, A. D., Darley, J. M. \& Cohen, J. D. The neural bases of cognitive conflict and control in moral judgment. Neuron 44, 389400 (2004)

73. Patil Indrajeet et al. Reasoning supports utilitarian resolutions to moral dilemmas across diverse measures. https://doi.org/10.31234/osf.io/q86vx. PsyArXiv (2018).

74. Miller, R. M., Hannikainen, I. A. \& Cushman, F. A. Bad actions or bad outcomes? Differentiating affective contributions to the moral condemnation of harm. Emotion 14, 573 (2014).

75. Lockwood, P. L. et al. Prosocial apathy for helping others when effort is required. Nat. Hum. Behav. 1, 0131 (2017).

76. Price, D. D. Psychological and neural mechanisms of the affective dimension of pain. Science 288, 1769-1772 (2000). 
1102 77. Will, G.-J., Rutledge, R. B., Moutoussis, M. \& Dolan, R. J. Neural and

1103 computational processes underlying dynamic changes in self-esteem. eLife 6,

$1104 \quad$ e28098 (2017).

1105 78. Railton, P. Moral Learning: Conceptual foundations and normative relevance.

$1106 \quad$ Cognition 167, 172-190 (2017).

1107 79. Nagel, T. What is it like to be a bat? Philos. Rev. 83, 435-450 (1974).

1108 80. Harsanyi, J. C. Cardinal welfare, individualistic ethics, and interpersonal

1109 comparisons of utility. J. Polit. Econ. 63, 309-321 (1955).

1110 81. Bentham, J. An introduction to the principles of morals and legislation.

$1111 \quad$ (Clarendon Press, 1879).

1112 82. Kant, I., Semple, J. W. \& Calderwood, H. The Metaphysic of Ethics. (T. \& T.

$1113 \quad$ Clark, 1871).

1114 83. Huys, Q. J. M. et al. Bonsai trees in your head: how the pavlovian system sculpts

1115 goal-directed choices by pruning decision trees. PLoS Comput. Biol. 8, e1002410

1116 (2012).

1117 84. Huys, Q. J. M. et al. Disentangling the Roles of Approach, Activation and

1118 Valence in Instrumental and Pavlovian Responding. PLOS Comput. Biol. 7,

1119 e1002028 (2011).

1120 85. Mackay, D. Model comparison and Occams Razor. Inf. Theory Inference Learn.

$1121 \quad$ Algorithms 343-355 (2003).

1122 86. Palminteri, S., Wyart, V. \& Koechlin, E. The Importance of Falsification in

1123 Computational Cognitive Modeling. Trends Cogn. Sci. 21, 425-433 (2017). 
1124 87. Eklund, A., Nichols, T. E. \& Knutsson, H. Cluster failure: Why fMRI inferences for

1125 spatial extent have inflated false-positive rates. Proc. Natl. Acad. Sci. 113, 7900

$1126 \quad$ (2016).

1127 88. Poldrack, R. A. et al. Guidelines for reporting an fMRI study. Neuroimage 40,

$1128 \quad$ 409-414 (2008).

1129 89. Mars, R. B. et al. Connectivity-Based Subdivisions of the Human Right

1130 'Temporoparietal Junction Area': Evidence for Different Areas Participating in

1131 Different Cortical Networks. Cereb. Cortex 22, (2012).

1132 90. Palomero-Gallagher, N. et al. Functional organization of human subgenual

1133 cortical areas: Relationship between architectonical segregation and

1134 connectional heterogeneity. Neurolmage 115, 177-190 (2015).

1135 91. Sallet, J. et al. The organization of dorsal frontal cortex in humans and

1136 macaques. J. Neurosci. 33, 12255-12274 (2013). 